\title{
Snapshot gradient-recalled echo-planar images of rat brains at long echo time at $9.4 \mathrm{~T}$
}

\author{
Hongxia Lei ${ }^{\mathrm{a}, \mathrm{b}, *}$, Vladimir Mlynárik ${ }^{\mathrm{a}}$, Nathalie Just ${ }^{\mathrm{a}, \mathrm{b}}$, Rolf Gruetter ${ }^{\mathrm{a}, \mathrm{b}, \mathrm{c}}$ \\ ${ }^{a}$ Laboratory for Functional and Metabolic Imaging, Ecole Polytechnique Fédérale de Lausanne, CH 1015 Lausanne, Switzerland \\ ${ }^{\mathrm{b}}$ Department of Radiology, University of Lausanne, CH 1015 Lausanne, Switzerland \\ ${ }^{\mathrm{c}}$ Department of Radiology, University of Geneva, CH 1205 Geneva, Switzerland \\ Received 30 November 2007; accepted 14 January 2008
}

\begin{abstract}
With improved $B_{0}$ homogeneity along with satisfactory gradient performance at high magnetic fields, snapshot gradient-recalled echoplanar imaging (GRE-EPI) would perform at long echo times (TEs) on the order of $T_{2}{ }^{*}$, which intrinsically allows obtaining strongly $T_{2}{ }^{*}$ weighted images with embedded substantial anatomical details in ultrashort time. The aim of this study was to investigate the feasibility and quality of long TE snapshot GRE-EPI images of rat brain at $9.4 \mathrm{~T}$. When compensating for $B_{0}$ inhomogeneities, especially second-order shim terms, a $200 \times 200 \mu \mathrm{m}^{2}$ in-plane resolution image was reproducibly obtained at long TE $(>25 \mathrm{~ms})$. The resulting coronal images at $30 \mathrm{~ms}$ had diminished geometric distortions and, thus, embedded substantial anatomical details. Concurrently with the very consistent stability, such GRE-EPI images should permit to resolve functional data not only with high specificity but also with substantial anatomical details, therefore allowing coregistration of the acquired functional data on the same image data set.
\end{abstract}

(C) 2008 Elsevier Inc. All rights reserved.

Keywords: Snapshot; Gradient-recalled echo-planar imaging; GRE-EPI; Rat brain; Long echo time; High field

\section{Introducton}

The significantly increased interest in rodent models for better understanding normal brain function and diseases drives demands for noninvasive methods. In vivo NMR allows noninvasively assessing brain metabolite concentrations, for example, neurochemical profile [1,2], cerebral blood flow [3,4], cerebral oxygen consumption rate [5] and cerebral glucose consumption rate [6-8]. Additionally, the capability of performing longitudinal studies and ultrashort acquisition times (such as EPI images) add to the uniqueness of in vivo NMR. With the recently increased interest in high magnetic field strength, the increased sensitivity allows to resolve physiological and pathological series, such as fMRI without averaging [9].

\footnotetext{
* Corresponding author. Ecole Polytechnique Fédérale de Lausanne, (EPFL), EPFL SB IPMC LIFMET, CH F1 627, Station 6, CH 1015, Lausanne, Switzerland. Tel.: +41 216937964; fax: +41 216937960.

E-mail address: hongxia.lei@epfl.ch (H. Lei).
}

However, most fMRI studies at high fields remained challenging due to some limiting factors, such as gradient performance, eddy currents, $B_{0}$ inhomogeneities and magnetic susceptibility artifacts. Both $B_{0}$ inhomogeneities due to magnetic susceptibility effects become more pronounced in rodent brains. This could be mainly explained by magnetic susceptibility effects that increase with reduced organ size. Unlike in human brain, the locally generated field gradients in rodent brains, especially nonlinear contributions, amplify with the correlation to the increasing brain surface-to-volume ratio leading to severe magnetic susceptibility artifacts at high fields [10]. The lack of capability of improving nonlinear-order $B_{0}$ homogeneities might have contributed to the limitation of high-quality in vivo NMR applications at higher fields by magnetic susceptibility effects for rodent brain imaging. Therefore, most gradient-echo (GE) EPI-based fMRI studies on rodent brains at high field have been limited to short echo time (TE) applications, such as snapshot gradient-recalled echo-planar imaging (GRE-EPI) with comparable spatial resolution [11], interleaved GRE-EPI [12], segmented GREEPI [13] and spin echo (SE)-EPI [14,15]. 
In the past 10 years, it has been shown that improved performance in gradients and in conjunction with several automatic shimming methods [16-18], $B_{0}$ inhomogeneities including nonlinear terms at high fields can be eliminated, leading to high-quality spectroscopy in rat or mouse brains $[1,2,19]$. From these studies, a $T_{2}^{*} \sim 30 \mathrm{~ms}$ was inferred from the achieved line width of the water signal of $10 \mathrm{~Hz}$. It is well established that the maximal relative signal changes due to $T_{2} *$ changes occur when $\mathrm{TE}=T_{2} *$ [20]. BOLD changes acquired at shorter TEs, for example, $10 \mathrm{~ms}$, would thus result in $\sim 60 \%$ of the maximum BOLD change. In addition to a potentially improved BOLD signal, acquiring EPI with longer TEs furthermore allows the collection of single-shot images, with concomitant gains in speed and reliability, compared to segmented or interleaved approaches [12].

We took delivery of a 9.4-T scanner with gradient coils with virtually absent eddy currents and ultrastrong shim coils, which suggested that high-resolution EPI with TE close to $T_{2}{ }^{*}$ was feasible. Therefore, the aim of the present study was to assess the feasibility and potential quality of high spatial resolution snapshot GRE-EPI images with long TE $(\sim 30 \mathrm{~ms})$ of rodent brain at $9.4 \mathrm{~T}$. A secondary aim was to evaluate the stability of GRE-EPI images for physiological studies.

\section{Methods}

\subsection{Animals}

All experiments were performed with approval from the local Animal Ethics Committee. Ten Sprague-Dawley male rats (Charles-River, France) with a mean weight of approximately $300 \mathrm{~g}$ were initially anesthetized under 5\% isoflurane in oxygen $\left(\mathrm{O}_{2}\right)$ and then maintained at $1.5-2 \%$ administered through a nose cone. Thereafter, each animal was stereotaxically fixed with two ear pieces and a bite bar in a homemade holder, which ensured placement of the animal head close to the isocenter of the magnet. Throughout the entire experiment, the animal was simultaneously monitored for breathing and temperature through an MR-compatible monitor system (SA Instruments, Model 1025, Stony Brook, NY, USA) and rectal temperature was maintained at $38.0^{\circ} \mathrm{C}$ by circulating warm water.

\section{2. $M R I$}

MRI experiments were performed in an actively shielded 9.4-T/31-cm horizontal magnet (Varian/Magnex Scientific, UK), which has a $B_{0}$ drift less than $10 \mathrm{~Hz} / \mathrm{h}$. The magnet was equipped with an actively shielded 12-cm-diameter gradient (400 $\mathrm{mT} / \mathrm{m}$ in $120 \mu \mathrm{s}$, Varian/Magnex Scientific) and custom-designed second-order shim coils with maximum strengths of $Z^{2}=5.3 \times 10^{-2} \mathrm{mT} / \mathrm{cm}^{2}, Y Z=1.2 \times 10^{-1} \mathrm{mT} / \mathrm{cm}^{2}$, $X Z=1.2 \times 10^{-1} \mathrm{mT} / \mathrm{cm}^{2}, X Y=4.5 \times 10^{-2} \mathrm{mT} / \mathrm{cm}^{2}$ and $X^{2} Y^{2}=4.2 \times 10^{-2} \mathrm{mT} / \mathrm{cm}^{2}$ (Varian/Magnex Scientific). The magnet was interfaced to a Unity INOVA console (Vnmrj, Varian Inc., Palo Alto, CA, USA). Eddy currents were minimized to be less than $0.01 \%$ using time-dependent quantitative eddy current field mapping [21]. A homemade quadrature ${ }^{1} \mathrm{H}$ radiofrequency (RF) coil with two geometrically decoupled 16-mm (inner diameter) loops resonating at $400 \mathrm{MHz}$ was used as RF transceiver.

Scout images were obtained using a GE sequence to determine appropriate positioning and shimming volume. Both first- and second-order shim coils were automatically adjusted over an $8 \times 6 \times 10 \mathrm{~mm}^{3}$ VOI using an EPI version of FASTMAP [16], which allowed to adjust all first- and second-order shim terms in less than $30 \mathrm{~s}$. In order to evaluate the resulting field homogeneity, an ultrashort echo time STEAM $[\mathrm{TE}=2 \mathrm{~ms}$, middle period $(\mathrm{TM})=20 \mathrm{~ms}]$ was applied to localize a $5 \times 5 \times 5 \mathrm{~mm}^{3}$ volume within the shimmed VOI.

Snapshot GRE-EPI traverses the entire $k$-space after one $\mathrm{RF}$ excitation. It is well established that any phase discrepancy due to the imperfection of gradient performance
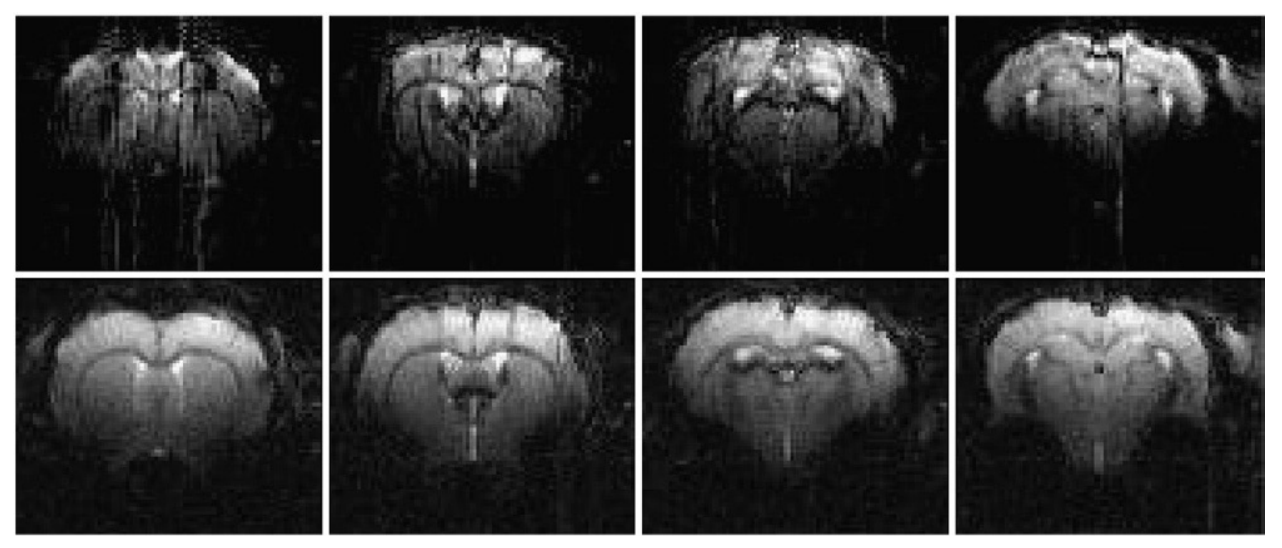

Fig. 1. Comparison of the snapshot GRE-EPI images acquired with both first- and second-order shim terms (the bottom row, selected from Panel A) and those with first-order shim terms only (the top row) on the identical slices from the same animal. In this particular study, the second-order shim terms, $Z^{2}=-0.49 \times 10^{-2}$ $\mathrm{mT} / \mathrm{cm}^{2}, Y Z=0.02 \times 10^{-2} \mathrm{mT} / \mathrm{cm}^{2}, X Z=-1.09 \times 10^{-2} \mathrm{mT} / \mathrm{cm}^{2}, X Y=-0.01 \times 10^{-2} \mathrm{mT} / \mathrm{cm}^{2}$ and $X^{2} Y^{2}=0.11 \times 10^{-2} \mathrm{mT} / \mathrm{cm}^{2}$, were applied before acquiring the images in the bottom row. 
Table 1

The applied averaged shim strengths and the corresponding maximum shim strengths of each second-order shim gradient at $9.4 \mathrm{~T}$

\begin{tabular}{lllll}
\hline Second-order shim gradient strength $\left(\times 10^{-2} \mathrm{mT} / \mathrm{cm}^{2}\right)$ & $Z^{2}$ & $Y Z$ & $X Y$ & $X^{2} Y^{2}$ \\
\hline System shim strength & 5.28 & 12.37 & 41.94 & 4.46 \\
Maximum absolute applied shim strength & 1.33 & 0.35 & 1.33 \\
Real applied shim strength & $-0.45 \pm 0.34$ & $-0.07 \pm 0.18$ & $-1.01 \pm 0.22$ & 0.08 \\
\hline
\end{tabular}

The applied second-order shim strengths were expressed as mean \pm S.D. $(n=10)$. Note that all the applied shim currents did not exceed $25 \%$ of the available maximum strength.

leads to Nyquist ghost artifacts. The built-in reference scan without phase-encoding gradient scheme [22] was applied to reduce the inconsistency between the odd and even echoes, which resulted in ghost artifacts of $\sim 2 \%$. Coronal images were acquired following a $2-\mathrm{ms} 90^{\circ}$ gauss-shaped excitation pulse with a native in-plane resolution of $200 \times 200 \mu^{2}$ (FOV $=25 \times 25 \mathrm{~mm}^{2}$ and $128^{2}$ data matrix) and TR/TE $=2000 /$ $30 \mathrm{~ms}$, whereas the phase gradient was applied along the $Y$ dimension. In order to avoid aliasing in the $Z$ dimension, transverse images were acquired with a slightly larger FOV of $40 \times 40 \mathrm{~mm}^{2}$. Concurrently with $\mathrm{TR} / \mathrm{TE}=2000 /$ $27 \mathrm{~ms}$, the $128^{2}$ data matrix resulted in a $312 \times 312 \mathrm{~mm}^{2}$ native in-plane resolution.

\subsection{Data analysis}

SNR was calculated from the most homogeneous and highest signal intensity region, which was in cortex due to the signal variation from using the surface coil as transmitter and receiver coil. Other ghosting and susceptibility artifacts were visually determined by comparing the GE and fast spin echo (FSE) images acquired with the identical position and slice thickness in the same rat. The stability of snapshot GRE-EPI images was determined by assessing the percent deviation of mean intensity for a region of interest (ROI) from 300 images of 2-mm slice thickness acquired with identical parameters over $10 \mathrm{~min}$. All data were analyzed with built-in imaging processing package (Vnmrj, Varian Inc.).

\section{Results}

Shortly, the EPI version of FASTMAP adjusted both firstorder and second-order shim strengths to compensate $B_{0}$ inhomogeneities over the whole rat brain region. In the present study, when adding second-order shims to the field inhomogeneity adjustment, which improved the water line width by only $1-2 \mathrm{~Hz}$, a noticeable effect on image quality was observed, as shown by comparing the acquisition of the identical slices with almost identical parameters after only first-order shimming was applied (Fig. 1). With the average applied magnitudes of second-order shim strengths (Table 1), the susceptibility artifacts and geometric distortion were more pronounced in the images acquired after compensating firstorder $B_{0}$ inhomogeneities only (Fig. 1, top row).

With applying second-order shim adjustment, the measured line widths of the water signal for the $5 \times 5 \times$ $5 \mathrm{~mm}^{3}$ volume were reduced to $11-13 \mathrm{~Hz}$, corresponding to an apparent $T_{2} *$ to $\sim 24-29 \mathrm{~ms}$. Consequently, the

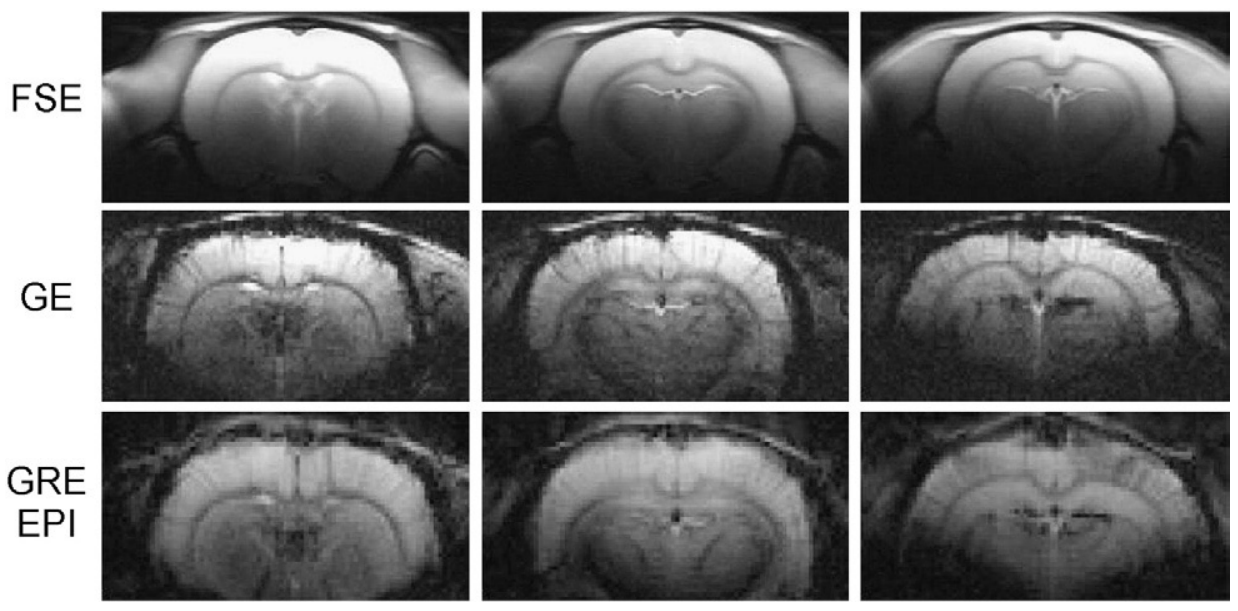

Fig. 2. Direct comparison between snapshot GRE-EPI, GE images and FSE images from one rat brain. All images were acquired after second-order shim adjustments with identical slice thickness of $1 \mathrm{~mm}$ and $0.5 \mathrm{~mm}$ gap. GRE-EPI and GE images were acquired using $200 \times 200 \mu \mathrm{m}^{2}$ in-plane resolutions, $30 \mathrm{~ms}$ TE and $2 \mathrm{~s}$ repetition time, but $30^{\circ}$ flip angle and $2.5 \mathrm{~ms}$ acquisition time (per $k$-space line in read dimension) were used in GE and $90^{\circ}$ flip angle and $0.3 \mathrm{~ms}$ acquisition time were used in GRE-EPI. The GRE-EPI images presented strong $T_{2}{ }^{*}$ contrast yet minor susceptibility artifacts, consistent with that present in GE images. 


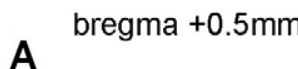

A

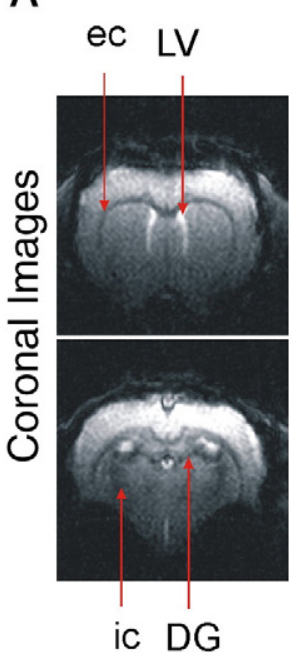

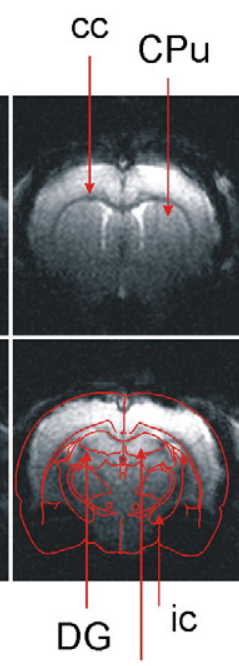

CA3
B

\section{bregma $-1.3 \mathrm{~mm}$}
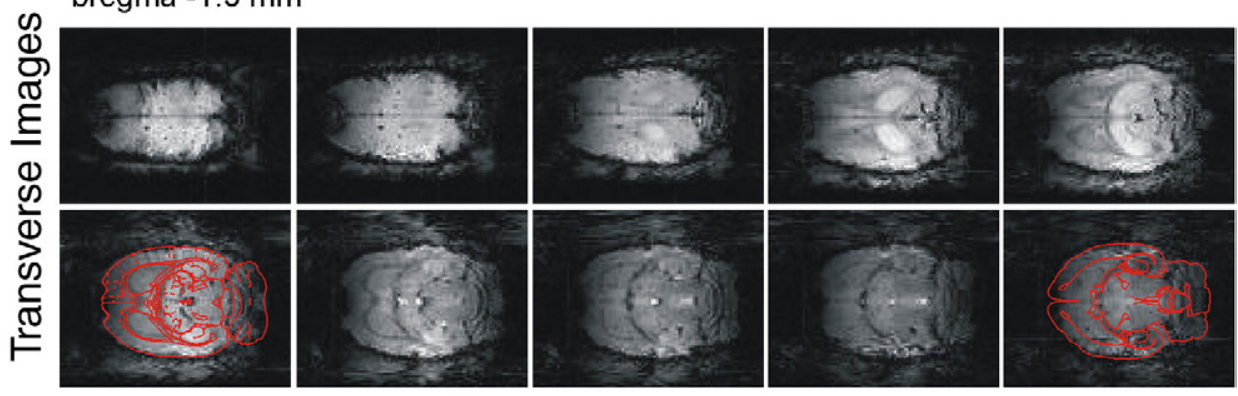

bregma $-6.8 \mathrm{~mm}$

Fig. 3. Multislice series of coronal (A) and transverse (B) GRE-EPI images acquired with TE $>20 \mathrm{~ms}$ at $9.4 \mathrm{~T}$. In Panel A, ten 0.5 -mm-thick slices without gap (from bregma $+0.5 \mathrm{~mm}$ to bregma $-3.8 \mathrm{~mm}$ ) were acquired with $200 \times 200 \mu \mathrm{m}^{2}$ in-plane resolution, TR/TE $=2000 / 30 \mathrm{~ms}$, as described in Section 2 . In Panel B, ten 0.5 -mm-thick slices with $312 \times 312 \mu \mathrm{m}^{2}$ in-plane resolution images (from bregma $-1.3 \mathrm{~mm}$ to bregma $-6.8 \mathrm{~mm}$ ) were acquired with the similar parameters as in Panel A but with $\mathrm{FOV}=40 \mathrm{~mm}^{2}$ and $\mathrm{TE}=27 \mathrm{~ms}$. Both images with long $\mathrm{TE}$ presented with less ghosting artifact and geometric distortions, which were further evidenced by being overlaid with the corresponding structures identified from the rat brain atlas (red lines in panels). Fine structures that were readily identified include 3V, CA3, cc, cg, CPu, D3V, DG, ec, ic, LV, MnPO, TS and vhc (see details in the text), indicated with red arrows in Panel A.

signal reductions and artifacts were reduced and snapshot GRE-EPI images were acquired at a comparable long TE, such as 30 ms with an increased spatial resolution (200× $200 \mu^{2}$ ). Minimal susceptibility effects were evident when comparing the GRE-EPI images to GE images acquired at the same TE (Fig. 2).

SNR ranged from 37 to 48 , assessed from a series of sixteen 0.5 -mm-thick multislice coronal images (Fig. 3A). These images presented well-resolved anatomical details on coronal (Fig. 3A) and transverse images (Fig. 3B). For example, the coronal images with a $200 \times 200 \mu \mathrm{m}^{2}$ in-plane resolution allowed identifying some fine structures such as the third ventricle (3V), CA3 of hippocampus (CA3), corpus callosum (cc), cingulum (cg), caudate putamen $(\mathrm{CPu})$, dorsal third ventricle (D3V), dentate gyrus (DG), external capsule (ec), internal capsule (ic), lateral ventricle (LV), median preoptic nucleus (MnPO), triangular septal nucleus (TS), ventral hippocampal commissure (vhc), and so forth. The reduced geometrical distortion in the images can be appreciated from the fact that without applying any distortion correction, the EPIs were superimposable on the rat brain atlas data (red lines in Fig. 3A and B).

Using a set of 2-mm-thick coronal images, we evaluated the stability of the time series from the percent deviation of mean intensity on the following ROIs: two pairs of 16-pixel (open diamonds and squares in Fig. 4A) and 45-pixel ROIs (open diamonds and squares in Fig. 4B), which was almost within $1.5 \%$ (peak-to-peak) over $10 \mathrm{~min}$. The percent deviations fell below $1 \%$ with the increased ROI to 32-pixel (two 16-pixel ROIs, filled circles in Fig. 4A), 45pixel (open diamonds and open squares in Fig. 4B) and 90pixel (the two 45-pixel ROIs, filled circles in Fig. 4B) ROIs. In order to determine if the signal variation was due to intrinsic SNR at such high spatial resolution or due to physiological fluctuations, we performed a linear regression analysis $(R=0.92)$ of the root-mean-square deviation 

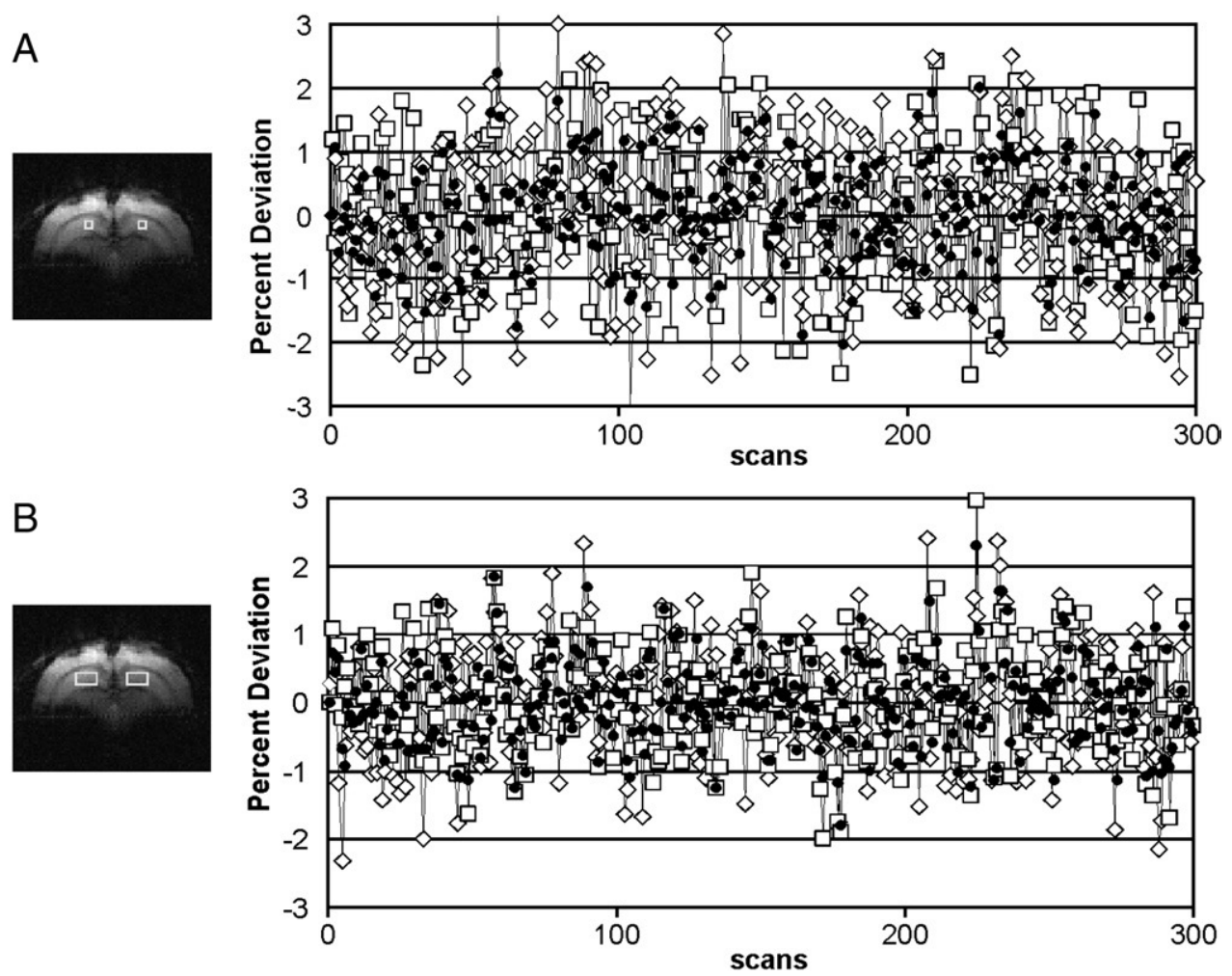

Fig. 4. Temporal stability of snapshot GRE-EPI images. The ROIs, in which the signals were measured, are marked in boxes on those $200 \times 200 \mu \mathrm{m}^{2}$ in-plane resolution and 1-mm slice thickness EPI images (A and B), which were acquired with two dummy scans and other parameters as described in Section 2. The corresponding percent deviations from the mean intensity from particular ROIs are shown as open squares for the left ROI and open diamonds for the right ROI. Filled circles denote both, respectively, on the side of each image. Over 10 min acquisition of $300 \mathrm{scans}$ ( $\mathrm{TR}=2 \mathrm{~s}$ ), the percent deviations are mostly within $1.5 \%$ for two individual 16-pixel ROIs in Image A. However, the deviations of both 16-pixel ROIs (the 32-pixel ROI) in Image A, two individual 45-pixel ROIs and both 45-pixel ROIs (the 90-pixel ROI) in Image B mostly fall in 1\%.

(RMSD) calculated from the RMSDs of the percent deviations, relative to inverse square root of the number of pixels (Fig. 5). The results indicated that a $Y$ intercept of $30 \%$ was present, which suggested that at this high resolution and long TE, noise was dominated by intrinsic image noise.

\section{RMSD}

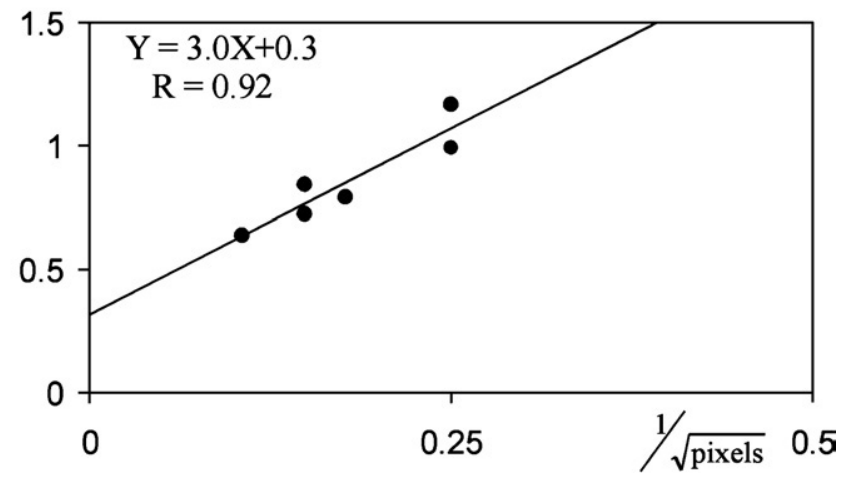

Fig. 5. Correlations between individual RMSD of each ROI with the corresponding volume from Fig. 4. A strong linear correlation $(R=.92)$ was found with $Y=3.0 X+0.3$, where $Y$ is the RMSD of the percent deviations and $X$ is $1 / \sqrt{\text { pixels, }}$ with "pixels" indicating the number of pixels of the corresponding ROI.

\section{Discussion}

The present study demonstrates that at $9.4 \mathrm{~T}$, snapshot GRE-EPI images with high in-plane resolution and long TE of rat brains can be reproducibly achieved (Figs. 1-4) with the combination of high performed gradients (rise time and gradient strengths), stable magnet $\left(B_{0}\right.$ drift), minimized eddy current and with compensated $B_{0}$ field inhomogeneities to second order. All of the above are likely to have contributed to the notable improvement and the very consistent stability of the GRE-EPI images shown in Fig. 4.

However, as illustrated in Fig. 1, such image quality with increased $T_{2}{ }^{*}$ and, thus, reduced signal dropout is unlikely to be achieved routinely without adjusting second-order shim terms. Concurrently, geometrical distortions were minimized as evidenced by comparison with the identical slice FSE images (Fig. 2) and with anatomical maps (red lines in Fig. 3).

It is of interest that two second-order shim terms, $Z^{2}$ and $X Z$ in Table 1, were shifted systematically to negative values with a significant range. This was not found in phantoms using the same setting and suggested to be the subject itself. Therefore, adjusting $B_{0}$ inhomogeneities, especially nonlinear-order terms, proves critical for rodent brains and, thus, obtaining quality GRE-EPI images at long TE. Overall, in the 
present study, the maximum requirements for the secondorder shim strengths to compensate the field inhomogeneities in the rodent brains were no more than $25 \%$ (Table 1). The average applied second-order shim strength for each shim coil was below $11 \%$ of the maximum of available shim strengths of the current system. Our strong customized shim strengths, thus, are likely capable of adjusting second-order shim terms in mouse brain [1], and thus, similar quality of snapshot GRE-EPI images of mouse brain with the identical in-plane resolution and TE should be possible.

The 30-ms TE used in the study is close to $T_{2}{ }^{*}$ reported from previous spectroscopy studies on rodent brains $[1,2,19]$ but slightly longer than the $T_{2}{ }^{*}$ in our observation $(\sim 24 \mathrm{~ms})$. This could be explained by the limited sensitivity of adjusting $B_{0}$ homogeneities over a larger VOI for imaging than for spectroscopy. However, $30 \mathrm{~ms}$ of TE used in the study remains in the range from 22 to $38 \mathrm{~ms}$, which allows to deliver $95 \%$ of the maximum BOLD change obtained at $\mathrm{TE}=T_{2}{ }^{*}[20]$. Even at such long $\mathrm{TE}$, the noise was mainly dominated by image noise not physiological noise (Figs. 4 and 5), which was reported to be mostly TE dependent [23]. Concurrently, the very consistent stabilities of the images (Fig. 4) were within the range reported from SE-EPI studies [14,24]. Therefore, such quality snapshot GRE-EPI images at high fields imply the ability to investigate the temporal evolution of fast physiological events, especially BOLD fMRI, which can be overlaid on simultaneously obtained anatomical information obtained from the same data set (Fig. 4), thereby eliminating coregistration issues typically encountered when combining fMRI with, for example, FSE anatomical images.

In the present study, we used the built-in traditional reference scan correction scheme [22] to reduce ghosting artifacts of the obtained GRE-EPI images. The remaining ghosting artifacts could be explained by uneven gradient performance with different polarities, suggested by other studies $[25,26]$. However, this would not change our observation that, other than the improved gradient performance, adjusting $B_{0}$ field inhomogeneities at high fields plays a very important role in obtaining highquality GRE-EPI images with high spatial resolution at long TE.

We conclude that high-quality snapshot GRE-EPI images of rat brain can be reproducibly obtained at $9.4 \mathrm{~T}$ at optimal TE for BOLD fMRI to allow a high spatial resolution with high anatomical contrast, eliminating coregistration issues.

\section{Acknowledgment}

The authors thank Dr. Ingrid Kohler and Hanne Frenkel for excellent technical supports. The work was supported by R01 NS042005 (NIH), the Centre d'Imagerie BioMédicale of the UNIL, UNIGE, HUG, CHUV and EPFL and the Leenaards and Jeantet Foundations.

\section{References}

[1] Tkac I, Henry PG, Andersen P, Keene CD, Low WC, Gruetter R. Highly resolved in vivo ${ }^{1} \mathrm{H}$ NMR spectroscopy of the mouse brain at 9.4 T. Magn Reson Med 2004;52(3):478-84.

[2] Tkac I, Starcuk Z, Choi IY, Gruetter R. In vivo ${ }^{1} \mathrm{H}$ NMR spectroscopy of rat brain at $1 \mathrm{~ms}$ echo time. Magn Reson Med 1999; 41(4):649-56.

[3] Silva AC, Kim SG, Garwood M. Imaging blood flow in brain tumors using arterial spin labeling. Magn Reson Med 2000;44(2): 169-73.

[4] Silva AC, Zhang W, Williams DS, Koretsky AP. Multi-slice MRI of rat brain perfusion during amphetamine stimulation using arterial spin labeling. Magn Reson Med 1995;33(2):209-14.

[5] Zhu XH, Zhang N, Zhang Y, Zhang X, Ugurbil K, Chen W. In vivo ${ }^{17} \mathrm{O}$ NMR approaches for brain study at high field. NMR Biomed 2005; 18(2):83-103.

[6] Sibson NR, Dhankhar A, Mason GF, Behar KL, Rothman DL, Shulman RG. In vivo ${ }^{13} \mathrm{C}$ NMR measurements of cerebral glutamine synthesis as evidence for glutamate-glutamine cycling. Proc Natl Acad Sci U S A 1997;94(6):2699-704.

[7] Choi IY, Lei H, Gruetter R. Effect of deep pentobarbital anesthesia on neurotransmitter metabolism in vivo: on the correlation of total glucose consumption with glutamatergic action. J Cereb Blood Flow Metab 2002;22(11):1343-51.

[8] Gruetter R, Adriany G, Choi IY, Henry PG, Lei H, Oz G. Localized in vivo ${ }^{13} \mathrm{C}$ NMR spectroscopy of the brain. NMR Biomed $2003 ; 16(6-7)$ : 313-38.

[9] Ugurbil K, Adriany G, Andersen P, Chen W, Gruetter R, Hu X, et al. Magnetic resonance studies of brain function and neurochemistry. Annu Rev Biomed Eng 2000;2:633-60.

[10] Grieve SM, Blamire AM, Styles P. The effect of bulk susceptibility on murine snapshot imaging at 7.0 T: a comparison of snapshot imaging techniques. Magn Reson Med 2000;43(5):747-55.

[11] Silva AC, Lee SP, Yang G, Iadecola C, Kim SG. Simultaneous blood oxygenation level-dependent and cerebral blood flow functional magnetic resonance imaging during forepaw stimulation in the rat. J Cereb Blood Flow Metab 1999;19(8):871-9.

[12] Guilfoyle DN, Hrabe J. Interleaved snapshot echo planar imaging of mouse brain at 7.0 T. NMR Biomed 2006;19(1):108-15.

[13] Huang W, Plyka I, Li H, Eisenstein EM, Volkow ND, Springer Jr CS. Magnetic resonance imaging (MRI) detection of the murine brain response to light: temporal differentiation and negative functional MRI changes. Proc Natl Acad Sci U S A 1996;93(12): $6037-42$.

[14] Chen Z, Shen J. Single-shot echo-planar functional magnetic resonance imaging of representations of the fore- and hindpaws in the somatosensory cortex of rats using an $11.7 \mathrm{~T}$ microimager. J Neurosci Methods 2006;151(2):268-75.

[15] Nair G, Duong TQ. Echo-planar BOLD fMRI of mice on a narrowbore 9.4 T magnet. Magn Reson Med 2004;52(2):430-4.

[16] Gruetter R, Tkac I. Field mapping without reference scan using asymmetric echo-planar techniques. Magn Reson Med 2000;43(2): 319-23.

[17] Hetherington HP, Chu WJ, Gonen O, Pan JW. Robust fully automated shimming of the human brain for high-field ${ }^{1} \mathrm{H}$ spectroscopic imaging. Magn Reson Med 2006;56(1):26-33.

[18] Shen J, Rothman DL, Hetherington HP, Pan JW. Linear projection method for automatic slice shimming. Magn Reson Med 1999;42(6): 1082-8.

[19] Mlynarik V, Gambarota G, Frenkel H, Gruetter R. Localized shortecho-time proton MR spectroscopy with full signal-intensity acquisition. Magn Reson Med 2006.

[20] Posse S, Wiese S, Gembris D, Mathiak K, Kessler C, Grosse-Ruyken ML, et al. Enhancement of BOLD-contrast sensitivity by single-shot multi-echo functional MR imaging. Magn Reson Med 1999;42(1): 87-97. 
[21] Terpstra M, Andersen PM, Gruetter R. Localized eddy current compensation using quantitative field mapping. J Magn Reson 1998; 131(1):139-43.

[22] Bruder H, Fischer H, Reinfelder HE, Schmitt F. Image reconstruction for echo planar imaging with nonequidistant $\mathrm{k}$-space sampling. Magn Reson Med 1992;23(2):311-23.

[23] Kruger G, Glover GH. Physiological noise in oxygenation-sensitive magnetic resonance imaging. Magn Reson Med 2001;46(4):631-7.
[24] Keilholz SD, Silva AC, Raman M, Merkle H, Koretsky AP. Functional MRI of the rodent somatosensory pathway using multislice echo planar imaging. Magn Reson Med 2004;52(1):89-99.

[25] Hu X, Le TH. Artifact reduction in EPI with phase-encoded reference scan. Magn Reson Med 1996;36(1):166-71.

[26] Chen NK, Wyrwicz AM. Removal of EPI Nyquist ghost artifacts with two-dimensional phase correction. Magn Reson Med 2004;51(6): 1247-53. 